\title{
Automatic Detection of Tap Changes on an Electricity Grid
}

\author{
Xiaoqing Chen \\ Dept. of Engineering Science \\ University of Oxford \\ Oxford, UK, OX1 3PJ \\ xiaoqing.chen@eng.ox.ac.uk
}

\author{
Stephen R. Duncan \\ Dept. of Engineering Science \\ University of Oxford \\ Oxford, UK, OX1 3PJ \\ stephen.duncan@eng.ox.ac.uk
}

\begin{abstract}
Demand side management (DSM) is a key concept for smart grid. In order to achieve DSM, network stress information needs to be provided to the users, which normally requires two-way communication between users and an upper level control centre. Implementing such communication in large scale power networks will be both costly and introduces problems with security and privacy of data. On-load tap changes (OLTC) are used to adjust the voltage at the users' side in response to network conditions. By detecting tap changes, an indication of the level of network stress can be provided to users without communicating with a control centre, which provides an economical and efficient measure for network stress. This paper presents a tap change detection algorithm based on Hilbert-transform phase-locked loops, Q-R decomposition windowed recursive least square algorithm and a hypothesis test, which can distinguish tap changes made by an OLTC transformer from the fluctuation in voltage caused by changes in loads. In this paper, the tap change detection algorithm is illustrated and the results for both simulated input and real-time data are presented and discussed.
\end{abstract}

\section{INTRODUCTION}

\section{A. POWER NETWORK AND DEMAND SIDE MANAGE- $M E N T$}

A conventional electric power system is a network that contains all the electrical components between generation and user [1]. A major issue for the existing electric power system is that it may not be able to meet the rapidly increasing demand for power. According to an estimate made by the International Energy Agency, global domestic energy demand will increase by $12 \%$ in 2020 compared to 2010 [2], whereas the power network capacity will only grow by $6 \%$ [3]. A conventional power system is a passive system which means it cannot adjust itself to react to the supply and demand condition [4], so in order to supply the demand which is uncontrollable and changing constantly, the capacity of the generation and the power system capacity must be designed to meet the maximum demand, and there should also be sufficient capacity remained for unpredictable demand. This is inefficient and by shifting demand from peak hour to other times, the efficiency of the power network can be improved [5]. One approach to achieve this is demand side management (DSM).

DSM is an important concept for smart grids, which modifies consumers' demand for energy via various methods, such as financial incentives [6]. For example, during peak hour, people can be encouraged to turn off appliances such as washing machine or air conditioner and turn them on again when it is off-peak time. Users can also use energy storage units to store energy during off-peak hours and discharge them during peak hours.

\section{B. STRESS OF LOCAL POWER NETWORKS}

In a power system, the supply and demand are constantly changing, so a mismatch always exists. The stress in a power system is defined by how close the demand is to the capacity of the operating limit [7]. Such a stress exists at both the national level and local level. At the national level, the imbalance between the generator and load will lead to the variation in the frequency of the sinusoidal voltage signal, whereas at local level, the stress is caused by the capacity of the power distribution cable. In this paper, we will concentrating on the local network stress.

Currently, much research has been done on identifying the local network stress, however most of the stress identification methods rely heavily on communication techniques such as ZigBee, WIFI or 3G/LTE [8], [9]. In these implementations, information from users is sent to a data concentrator and pre-processed, so that the network stress information will be sent back from the control centre to users. Achieving such a huge amount of communication requires a well-developed information network, which is both costly and vulnerable.

In a power distribution system, in order to balance the voltage loss along the cable and keep the voltage at the user side stable, on-load tap changer (OLTC) transformers are used. An OLTC transformer reacts to different load conditions by moving on tap to different winding contacts, adjusting its transformer ratio and changing its output voltage, which is called tap change. Tap changes are operated according to the variation of local network stress. As tap changes can be detected by measuring magnitude change of the $230 \mathrm{~V}$ voltage signal which is accessible to the users, this can be exploited to allow local users to detect changes in the stress of the network. It is more convenient to identify network stress by detecting tap changes in this way because communication with the data concentrator is not required.

The paper is structured as follows. Section 2 introduces three main components of the tap change detection algorithm and tests them with simulated input. Section 3 presents and discusses results of the algorithm with real-time input data which is obtained from a power network. The conclusion is given in section 4 . 


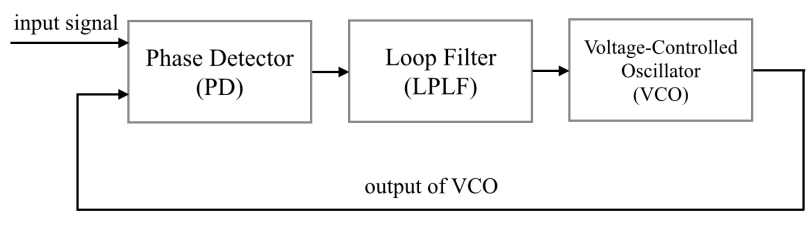

Fig. 1. Diagram of PLL [11]

\section{STRESS IDENTIFICATION TECHNIQUE}

An algorithm for network stress detecting is introduced in this section. In this algorithm, the frequency of the sinusoidal voltage signal is estimated by a phase-locked loop (PLL). Given an estimate of the frequency, the magnitude of the voltage is calculated by the windowed recursive least square (WRLS) algorithm. A hypothesis test is used for detecting whether a magnitude changes is caused by noise or caused by a tap change.

\section{A. FREQUENCY TRACKING ALGORITHM}

There are several methods for finding frequency of a periodic signal including zero crossing detection and phaselocked loops (PLL) [10]. Given that a PLL can track frequency changes quickly and also handle with signal with high signal to noise ratio (SNR), a PLL is chosen as the frequency tracking unit in our algorithm.

A PLL is a circuit that can generate an output signal with the same frequency as an input or reference signal. As shown in Fig. 1, there are three components of a PLL, they are phase detector (PD), loop filter (LF) and voltage-controlled oscillator (VCO) [12], [13]. In our implementation, the PLL is Hilberttransform phase-locked loop (HTPLL), which uses a Hilberttransformer phase detector (HTPD). A HTPLL rather than a conventional PLL is chosen because the phase detector used by a conventional PLL is simply a multiplier, which generates a second order harmonic component in its output, whereas HTPD used by HTPLL is based on the Hilbert-transform, which delays the phase of the input signal for $\pi / 2$ [14]. As a result, a HTPLL will not generate the second order harmonic component in the output and thus can provide better performance.

In HTPLL, the input signal is applied to both a Hilbert transformer and a delay function, thus we can have $A_{i} \cos \left(2 \pi f_{i} t+\right.$ $\left.\theta_{i}\right)$ and $A_{i} \sin \left(2 \pi f_{i} t+\theta_{i}\right)$. From the VCO, we can obtain $A_{o} \cos \left(2 \pi f_{o} t+\theta_{o}\right)$ and $A_{o} \sin \left(2 \pi f_{o} t+\theta_{o}\right)$ easily. Define $\phi_{e}=$ $\phi_{i}-\phi_{o}$, then

$$
A \cos \phi_{e}=A \cos \left(\phi_{i}-\phi_{o}\right)
$$

where $A=A_{i} A_{o}$, so

$$
A \cos \phi_{e}=A_{i} \cos \phi_{i} \cdot A_{o} \cos \phi_{o}+A_{i} \sin \phi_{i} \cdot A_{o} \sin \phi_{o}
$$

Similarly,

$$
A \sin \phi_{e}=A_{i} \sin \phi_{i} \cdot A_{o} \cos \phi_{o}-A_{i} \cos \phi_{i} \cdot A_{o} \sin \phi_{o}
$$

so that

$$
S_{e}(t)=\tan ^{-1} \frac{A \sin \phi_{e}}{A \cos \phi_{e}}=\left(\phi_{i}-\phi_{o}\right) \bmod (2 \pi)
$$

In this way, $S_{e}$ which is the output of HTPD, is the phase error between the input signal of PLL and the output signal of the VCO.

The high frequency noise in $S_{e}$ is smoothed by a loop filter. In order to make the HTPLL to be a second order 'Type 1' system so that it can achieve zero steady-state error, the LF should have at least one integrator. So the simplest LF is a PI controller [11], whose transfer function is

$$
C(s)=K\left(1+\frac{1}{s T_{i}}\right)
$$

The closed-loop transfer function from the input phase to the output phase of the PLL with PI controller is

$$
G_{e}(s)=\frac{\frac{K N}{2 T_{i}}+s}{s^{2}+\frac{K N}{2} s+\frac{K N}{2 T_{i}}}
$$

where $N$ is the gain of the phase detector. For HTPD, the phase detector gain is $N=1$. Comparing (6) with the standard second order transfer function, it can be obtained that the natural frequency for HTPLL with PI controller is

$$
\omega_{n}=\sqrt{\frac{K N}{2 T_{i}}}
$$

with damping factor

$$
\zeta=\sqrt{\frac{K N T_{i}}{8}}
$$

where $\omega_{n}$ and $\zeta$ are design parameters. In order to decrease the overshoot and achieve good tracking, we choose $\zeta=1$, so that,

$$
\begin{aligned}
K & =\frac{4 \omega_{n}}{A} \\
T_{i} & =\frac{2}{\omega_{n}}
\end{aligned}
$$

The output of LF can be directly used to control the VCO. With the coordination of HTPD, LF and VCO, the PLL can generate an output signal with the same frequency as its input.

Fig. 2 shows the simulation result of HTPLL with a step change in the input signal frequency from $50 \mathrm{~Hz}$ to $51 \mathrm{~Hz}$. It can be seen from the figure that the HTPLL reacts to a step change in the input frequency and tracks the new input frequency quickly and it can also be noticed that even though the damping factor is $\zeta=1$, due to the effect of the zero in the close loop transfer function (6), there is still an overshoot in the output frequency.

\section{B. TAP CHANGE DETECTION ALGORITHM}

In a power network, there are numerous loads operating and there is noise as well. These disturbances, combined with tap changes, cause the magnitude of the voltage signal to be constantly fluctuating. For distinguishing tap changes from voltage fluctuation caused by noise, the magnitude of the sinusoidal signal needs to be known first, and then a hypothesis test can be used to check tap changes, so there 


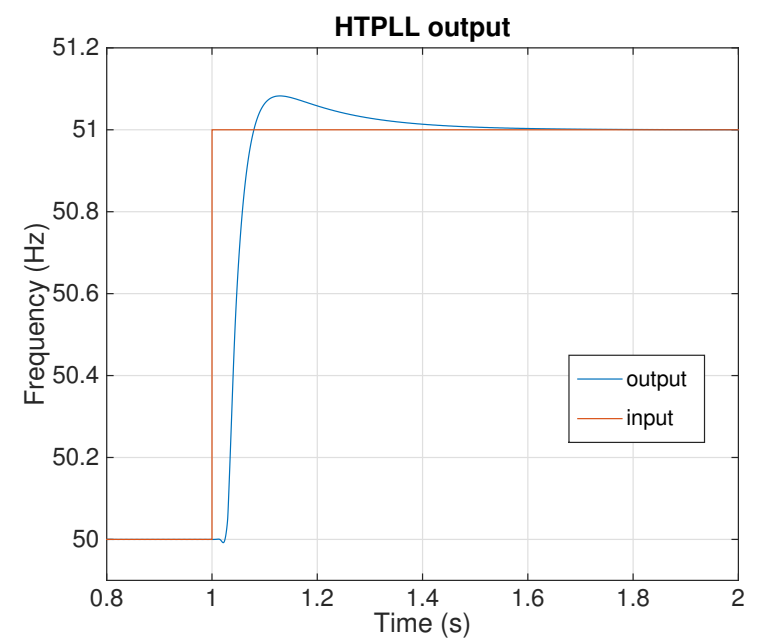

Fig. 2. HTPLL reaction to a step change in input frequency

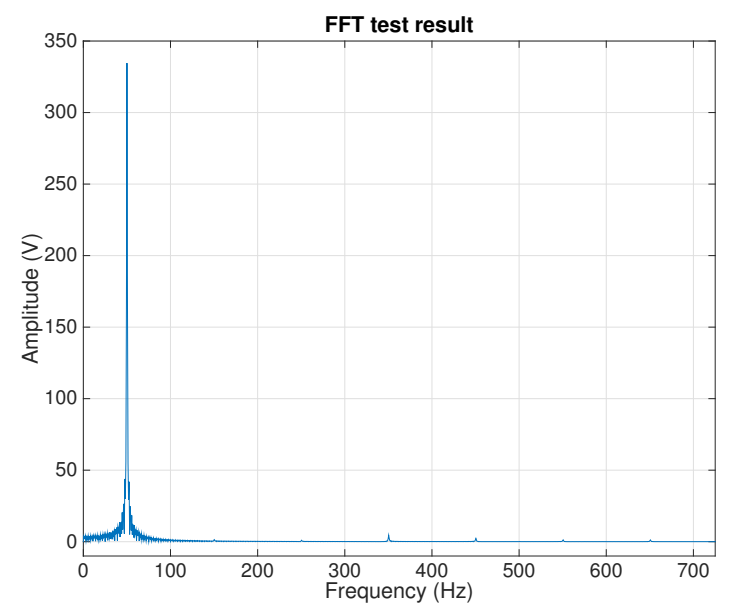

Fig. 3. FFT result

are two parts in the tap change detection algorithm. The first part is a windowed recursive least squares algorithm, which is implemented using the Q-R decomposition (QRD-WRLS). The second part is a hypothesis test for the fluctuation of the signal amplitude which is operating within the sliding window.

1) $Q R D$-WRLS ALGORITHM: Due to the saturation effect of the transformer and many other disturbances, the voltage signal in power grid is not a pure sinusoid wave but contains high harmonic contents.

The periodic voltage signal can be expressed as the following format

$$
y_{i}=\sum_{k=1}^{n} A_{k} \cdot \cos \varphi_{k}(t)
$$

where $k$ is the order of the harmonic, $A_{k}$ is the amplitude of the $k^{t h}$ harmonic signal, $t$ is time and $\varphi_{k}$ is the phase of the $k^{\text {th }}$ harmonic signal which can be interpreted as

$$
\begin{aligned}
\varphi_{k}(t) & =2 \pi \int_{0}^{t} k f_{0}\left(t^{\prime}\right) d t^{\prime} \\
\varphi_{k}(0) & =\theta_{k}
\end{aligned}
$$

where $f_{0}(t)$ is the fundamental frequency. Fig. 3 shows the FFT result of real-time voltage signal recorded from power grid. It can be seen from the figure that there are high harmonic contents in the signal, but it is obvious that most power is concentrating on the fundamental harmonic. The magnitude of the high harmonics are negligible comparing to that of the fundamental harmonic. So the expansion in (11) can be rearranged as

$$
y=a_{1} \cos \left(\varphi_{1}(t)-\theta_{1}\right)+b_{1} \sin \left(\varphi_{1}(t)-\theta_{1}\right)
$$

where $\tan \theta_{1}=-b_{1} / a_{1}$, and $a_{1}, b_{1}$ are the amplitude of the sine and cosine components of the fundamental harmonic. The amplitude of the signal, when the high harmonic content is ignored, can be calculated by the following equation

$$
A_{1}=\sqrt{a_{1}^{2}+b_{1}^{2}}
$$

An estimate of phase $\varphi_{1}(t)$ in (14) can be obtained from the HTPLL, so we can use the QRD-WRLS algorithm to obtain $a_{1}$ and $b_{1}$, and then use (15) to calculate the magnitude of the signal.

The reason for using QRD-WRLS algorithm rather than normal windowed recursive least square algorithm is that QRD-WRLS is numerically robust which means it can provide the more reliable results than the normal method [15], [16]. There are many approaches to implement QRD-WRLS, but the one proposed by Yoo and Park [17], is used in this algorithm.

Fig. 4 shows the simulation result of QRD-WRLS algorithm with a simulated sinusoidal voltage input. There is a step change in the amplitude of the voltage signal at $t=0.5 \mathrm{~s}$. The simulated input signal was first put into HTPLL so that the phase information can be obtained. Then the voltage signal along with its phase information was processed by QRDWRLS algorithm.

The figure shows that the QRD-WRLS algorithm can calculate the magnitude accurately and when the magnitude of the signal is changed, the algorithm reacts to the change quickly.

2) HYPOTHESIS TEST: A segment of the voltage signal containing a tap change is shown in Fig. 5 (only the top section of the sinusoidal voltage signal is shown). In the figure, the amplitude of the voltage signal in power distribution system before and after a tap change is different, so a hypothesis test approach can be used to detect whether this is due to a tap change. A suitable hypothesis test for tap change detection is the generalized likelihood ratio (GLR) test.

Likelihood is a measure of likeliness of what has been observed when given a certain assumption. On the basis of observed data, the likelihood of different assumptions on at which a change in amplitude occurred can be compared [18], [19]. For a model such as

$$
y_{t}=\theta+e_{t}, \quad e_{t} \in N(0, R)
$$




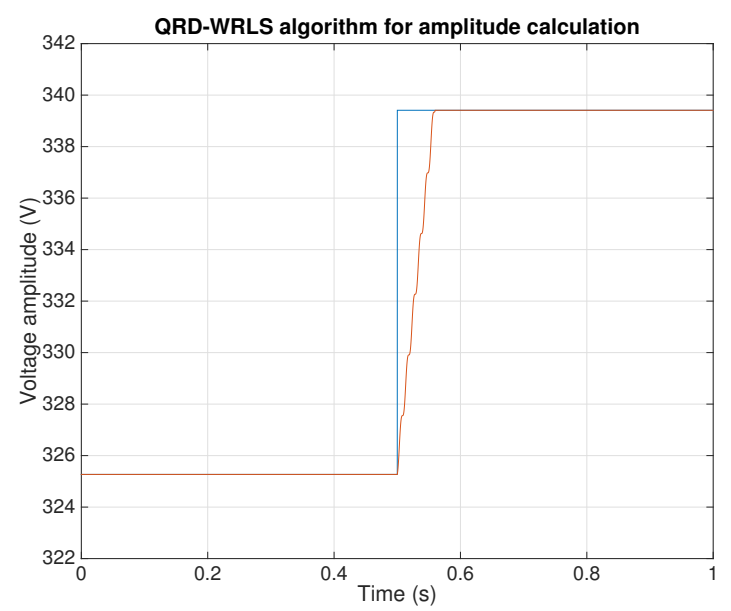

Fig. 4. QRD-WRLS algorithm result for amplitude calculation

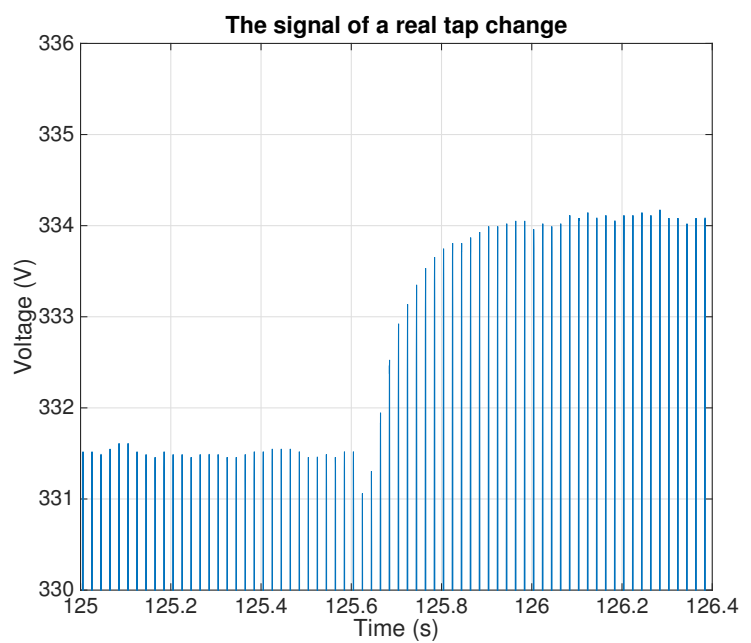

Fig. 5. Amplitude change for a real tap change

where the noise follows a Gaussian distribution, then based on generalized likelihood estimation, the log-likelihood for the case where the mean value $\theta$ and the noise variance $R$ is known, given [18]

$$
-2 \log \left[l_{t}^{M G L}(R)\right] \approx t \log (2 \pi R)+\frac{t \hat{R}}{R}
$$

where $\hat{R}$ is the actual variance of the data set $y_{1}, y_{2}, \ldots, y_{t}$. Assuming that the values of $\theta$ before and after the change are independent, then the likelihood can be divided into two parts,

$$
p\left(y^{t} \mid k\right)=p\left(y^{k} \mid k\right) p\left(y_{k+1}^{t} \mid k\right)=p\left(y^{k}\right) p\left(y_{k+1}^{t}\right)
$$

and the likelihood ratio can be used to compare the likelihood of null hypothesis, against the change hypothesis.

$$
H_{0}: \text { no change happens }
$$

$H_{1}(k, v)$ : a jump of magnitude $v$ at time $k$
The log likelihood ratio for the hypothesis test is

$$
g_{t}(k, v) \triangleq 2 \log g_{t}^{G}(k, v)=2 \log \frac{p\left(y^{t} \mid H_{1}(k, v)\right)}{p\left(y^{t} \mid k=t\right)}
$$

where $g_{t}$ is the log likelihood ratio and $g_{t}^{G}$ is the likelihood ratio. With (18) and (19) the GLR statistic can be calculated.

The classical GLR method is an off-line algorithm. For the problem in this paper, however, tap changes can happen at any time and as time evolves, new data needs to be included. To accommodate this, the generalized likelihood test is applied over a sliding window. For the arrangement of the sliding window, a sliding window $W_{L}$ with the length of $2 d$ is set in advance. $W_{L}$ is equally divided into two smaller windows $W_{1}$ and $W_{2}$, so that $W_{1}$ and $W_{2}$ contain equal number $d$ data. The GLR result for the data in $W_{L}$ can be expressed as

$$
g_{t}\left(W_{L}\right)=2 \log \frac{l_{W_{1}}^{M G L} \cdot l_{W_{2}}^{M G L}}{l_{W_{L}}^{M G L}}
$$

The statistic in (20) can be calculated using (17), and the variance $\hat{R}$ in (17) can be calculated using $\hat{R}=\overline{y^{2}}-\bar{y}^{2}$. It is straightforward to update this algorithm by updating and downdating the mean $\bar{y}$ and quadratic mean $\overline{y^{2}}$ of the data in these windows. Every time new data arrives, the sliding window is moved forward and the parameters of the sliding windows will be updated and downdated. The GLR result $g_{t}\left(W_{L}\right)$ for the sliding window indicates the probability of change happening at the mid point of it.

The GLR test statistic $g_{t}^{G}$ follows the $\chi^{2}$ distribution, and so the distribution of $g_{t}$ can be obtained using (19). The tap change detection algorithm is designed to operate with a huge amount of data. In order to achieve high reliability, the confidence interval for the GLR test threshold $h_{g}$ need to be higher than $99.99 \%$. The test threshold for the log generalized likelihood ratio is $h_{L}=2 \log h_{g}$. An improved threshold can be obtained by considering the feature of tap change outlined in Fig. 5. If a tap change leads to a $V_{c}$ volt voltage change in $T_{c}$ seconds and the lengths $d$ of the moving data windows $W_{1}$, $W_{2}$ are small enough, and data is sampled with the sampling frequency of $f_{s} \mathrm{~Hz}$. We assume that the voltage is increasing linearly and there is no noise during the tap change, the GLR value of the tap change can be calculated with (20), and it can be rearranged as

$$
g_{t, \text { min }}=\frac{V_{c}^{2} d^{2}(3 d-2)}{6 T_{c}^{2} f_{s}^{2} R}
$$

From (17) it can be concluded that when there is voltage fluctuation, the variance of the data will be larger. Therefore the test statistic will be larger than $g_{t, \min }$. As such $g_{t, \min }$ can be used as the test threshold as long as it can promise the confidence interval to be higher than $99.99 \%$. Actually, compared to the variance of the voltage magnitude $R$, the voltage change $V_{c}$ during tap change is very large and hence the confidence interval requirement can be easily satisfied. For example, for real time data, when we set the noise variance $R=0.3 \mathrm{~V}$, the threshold $h_{L}$ for $99.99 \%$ is 12.8 , whereas $g_{t, \text { min }}$ 


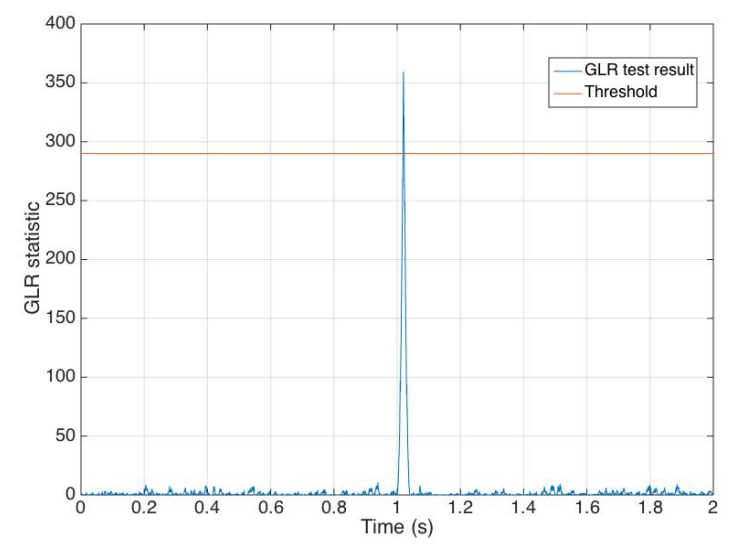

Fig. 6. Simulation result for GLR test

is around 280. Therefore it is reasonable to use $g_{t, \min }$ as the test threshold.

Fig. 6 shows the simulation result of the GLR test. The input is a simulated voltage signal and there is an amplitude change of $2 \mathrm{~V}$ in the input signal at time $t=1 \mathrm{~s}$. It can be seen from the figure that the test statistic was fluctuating when there is no amplitude change and the value was always below the threshold. However when there is an amplitude change in the input, the test result will increase abruptly and exceed the threshold. In the figure the GLR result exceeds the threshold shortly after $t=1 \mathrm{~s}$ whereas we know from the input that the change happens exactly at $t=1 \mathrm{~s}$. This time delay is caused by the length of the sliding window.

\section{RESULT AND DISCUSSION}

\section{A. FREQUENCY}

In this section, the results of using this algorithm with real-time data from a power network will be presented and discussed. The real-time voltage data was obtained from a data logger, with the sampling frequency of $f_{s}=10 \mathrm{kHz}$. It recorded the mains voltage signal of one phase over the period from 12th March 2014 to 14th March 2014.

Using the frequency tracking algorithm, the variation of the signal frequency can be obtained. Fig. 7 shows the frequency change over 24 hours on the 12th of March, 2014, where the mean frequency is plotted every 10 minutes. It can be seen that the frequency of the signal was changing although the fluctuation of the frequency is approximately within $\pm 0.1 \mathrm{~Hz}$ or $0.2 \%$, which is acceptable with the national regulation of $1 \%$. This fluctuation of the frequency is caused the variation of the mismatch between the supply side and demand side of the power grid.

\section{B. AMPLITUDE}

Fig. 8 shows the output of the combination of HTPLL and QRD-WRLS algorithm with real-time voltage input. In the figure, the top portion of the input can be seen, and the line

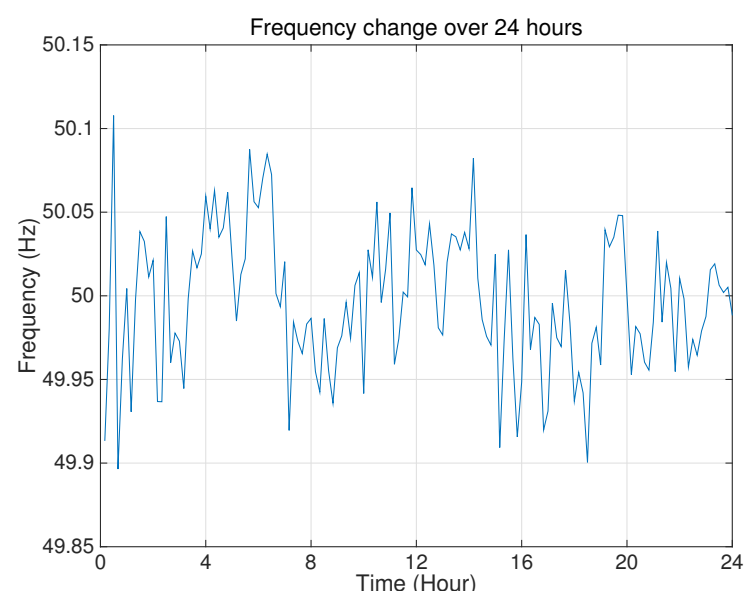

Fig. 7. Frequency change over 24 hours

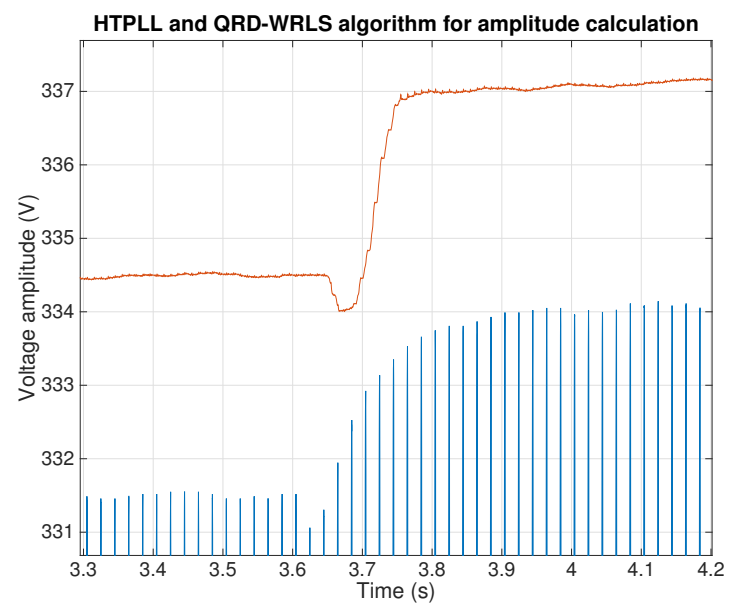

Fig. 8. Estimated amplitude (red) and top section of voltage fluctuation (blue)

at the upper part is output of QRD-WRLS algorithm, which is the estimate of the input signal's amplitude.

It can be seen from the figure that the output of the algorithm can provide an accurate estimate of the magnitude of the sinusoidal voltage signal. Also, there is a tap change in the input signal in Fig 8 and the output reflects the magnitude change of the tap change. As mentioned in previous sections, there is also high harmonic contents in the input signal, although in QRD-WRLS algorithm only the fundamental harmonic is calculated. As a result, the estimated magnitude is slightly larger than the magnitude of the real-time input. There is also a time delay in the output compared to the input, which is due to the length of the sliding windows of the algorithm.

\section{HYPOTHESIS TEST}

Fig. 9 shows the typical output of the combined algorithm with real-time tap change input data (the top part of the input is shown in Fig 5 and it can be seen that a tap change happens at about $t=125.6 \mathrm{~s}$ ). It can be seen from the figure that, the GLR 


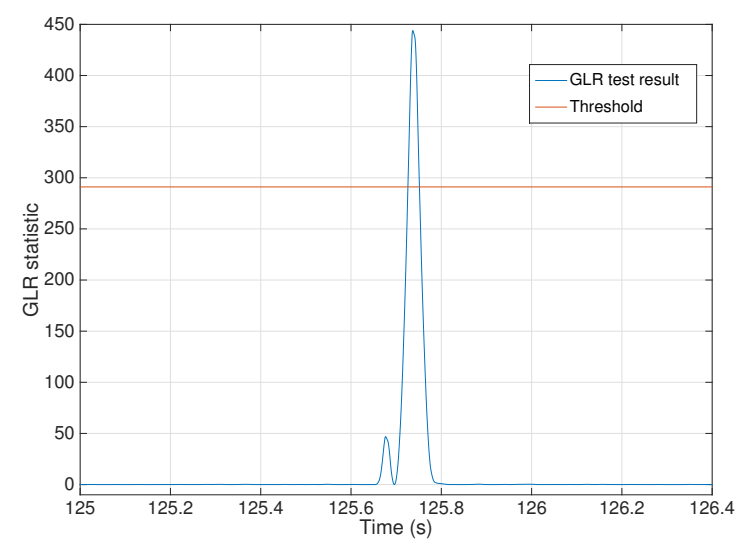

Fig. 9. GLR result with real tap change input

result is quite small and fluctuating within a negligible range when there is no tap change. However, when a tap change occurs, the GLR value increases abruptly to a large level and exceed the threshold (at about $t=125.7 \mathrm{~s}$, the $0.1 \mathrm{~s}$ time delay is due to the length of the sliding window).

TABLE I shows the tap change detection result with the three days' voltage input data of the peak hours of consumption (the peak hours start from 6:00 to 10:00 and 17:00 to 24:00). All the tap changes happened in the three days were occurred during the time the data covered, indicating that the load change and voltage fluctuation is comparatively severe in this period.

TABLE I

REAL TIME RESULT FOR GLR TEST

\begin{tabular}{ccc}
\hline Time & Output tap change time & Correct detection \\
\hline \multirow{4}{*}{ 12 March 2014} & $06: 56: 17$ & $\sqrt{ }$ \\
& $08: 09: 51$ & $\times$ \\
& $09: 30: 39$ & $\times$ \\
$17: 26: 06$ & $\sqrt{ }$ \\
$21: 09: 57$ & $\sqrt{ }$ \\
& $22: 48: 38$ & $\sqrt{ }$ \\
& $07: 02: 38$ & $\times$ \\
13 March 2014 & $08: 23: 30$ & $\sqrt{ }$ \\
& $09: 07: 34$ & $\times$ \\
& $09: 08: 30$ & $\times$ \\
& $09: 12: 40$ & $\sqrt{ }$ \\
& $22: 11: 52$ & $\sqrt{ }$ \\
\hline \multirow{5}{*}{ 14 March 2014} & $23: 10: 35$ & $\sqrt{ }$ \\
& $06: 38: 26$ & $\times$ \\
& $08: 08: 21$ & $\times$ \\
& $08: 11: 07$ & $\sqrt{ }$ \\
& $17: 24: 10$ & $\sqrt{ }$ \\
\hline
\end{tabular}

Comparing the result to the record of the OLTC transformer, it can be seen that all the tap changes are successfully detected by the algorithm, however, there are also some incorrect detection results. In the real input data, the magnitude of the voltage sometimes fluctuates strongly (a typical voltage

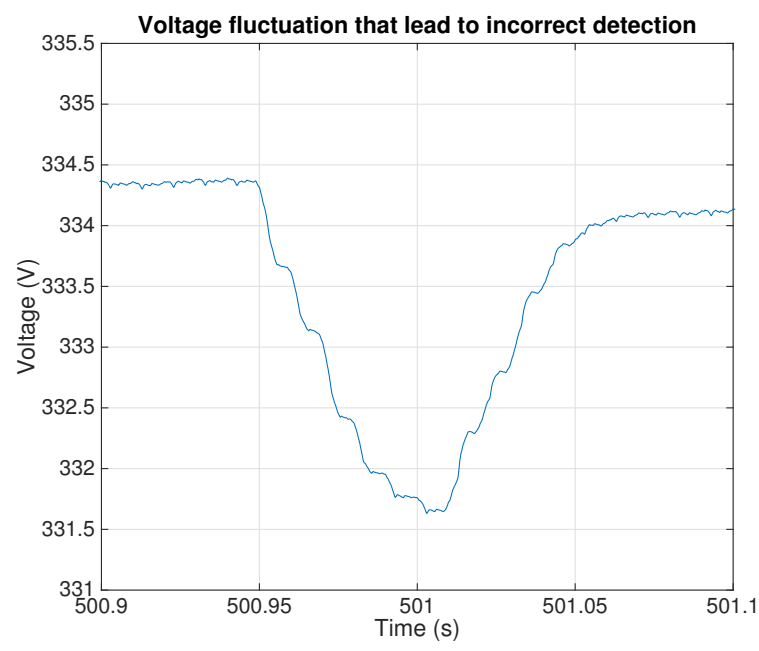

Fig. 10. Voltage fluctuation

magnitude fluctuation curve is shown in Fig. 10). For such strong voltage fluctuations, their GLR statistic will be very large and exceed the threshold, so the algorithm regards these fluctuations as tap changes. From Fig. 10, we can see that

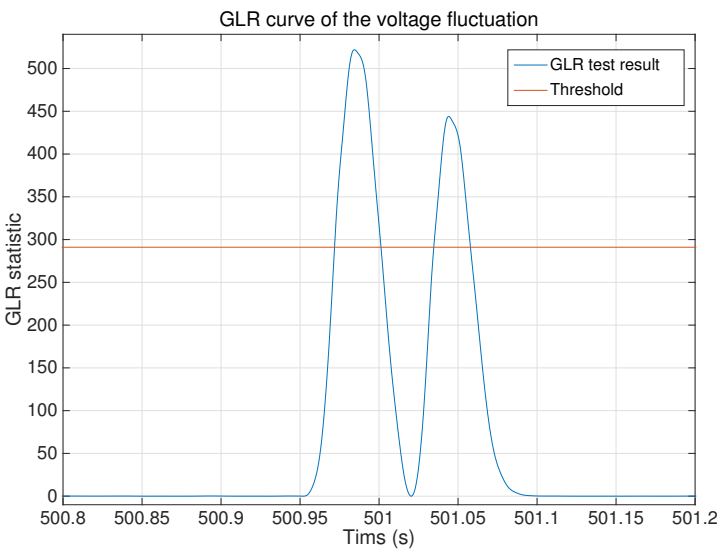

Fig. 11. GLR curve of the voltage fluctuation

for such voltage fluctuation, the voltage first drops for about $2.5 \mathrm{~V}$ in about $0.06 \mathrm{~s}$ and then rises again to the previous level quickly. The changing trend for such voltage fluctuations is different from that of the tap changes, as the magnitude will go back to the previous level quickly rather than goes up or drops down directly. Therefore, these voltage fluctuations have different GLR curves from that of tap changes.

The GLR curve for the voltage fluctuation in Fig. 10 is shown in Fig. 11. Comparing Fig 11 and Fig. 9, we can see that the GLR curve of voltage fluctuation and tap change is different. A tap change will just lead to one pulse in the GLR result, but a voltage fluctuation will lead to two pulses in the GLR result, which can be used to identify voltage fluctuation form the tap change. As this feature is added into 
tap change identification, the incorrect detections in TABLE I can be corrected. In this way, tap changes can be effectively distinguished from noise and voltage fluctuation.

\section{CONCLUSIONS}

This paper considers stress identification for DSM via tap change detection. The tap change detection algorithm introduced in this paper provides a stress identification method which can be used to indicate local network stress directly from the user side without communication to a control centre. There are three main sections of the algorithm: a HTPLL is used for finding the frequency of the sinusoidal voltage signal, QRD-WRLS algorithm is used for finding the amplitude of the signal and hypothesis test is used for testing tap changes from the variation of the voltage amplitude.

From the results with simulated and real-time input data, it is shown that the algorithm can track the frequency of the sinusoidal voltage signal and find the amplitude of the signal and tap changes are detected by the GLR hypothesis test. It can also be seen that this algorithm can provide good result for tap change detection which can be used to indicate local network stress.

\section{ACKNOWLEDGEMENT}

The real-time voltage data are provided by Professor Malcolm D. McCulloch and Ren Kang from the Energy and Power Group of the Department of Engineering Science, University of Oxford, which were obtained from Scottish and Southern Energy (SSE) plc., Reading.

\section{REFERENCES}

[1] C. Rey. Superconductors in the Power Grid : Materials and Applications. Amsterdam : Elsevier : Woodhead Publishing, 2015.

[2] International Energy Agency. Cool Appliances: Policy Strategies for Energy-Efficient Homes. OECD Publishing, 2003.

[3] N. Gudi, L. Wang, and V. Devabhaktuni. A demand side management based simulation platform incorporating heuristic optimization for management of household appliances. International Journal of Electrical Power \& Energy Systems, 43(1):185-193, 2012.

[4] P. J. Agrell, P. Bogetoft, and M. Mikkers. Smart-grid investments, regulation and organization. Energy Policy, 52:656-666, 2013.

[5] G. Strbac. Demand side management: Benefits and challenges. Energy Policy, 36(12):4419-4426, 2008.

[6] D. Geelen, A. Reinders, and D. Keyson. Empowering the end-user in smart grids: Recommendations for the design of products and services. Energy Policy, 61:151-161, 2013.

[7] R. Kang and M. McCulloch. Identification of electric power system stress through feeder voltage measurement. In Innovative Smart Grid Technologies Conference Europe (ISGT-Europe), 2014 IEEE PES, pages 1-6, Istanbul, Turkey, 2014. IEEE.

[8] Z. Fan, P. Kulkarni, S. Gormus, C. Efthymiou, G. Kalogridis, M. Sooriyabandara, Z. Zhu, S. Lambotharan, and W. H. Chin. Smart grid communications: overview of research challenges, solutions, and standardization activities. Communications Surveys \& Tutorials, IEEE, 15(1):21-38, 2013.

[9] V. C. Güngör, D. Sahin, T. Kocak, Sa. Ergüt, C. Buccella, C. Cecati, and G. P. Hancke. Smart grid technologies: communication technologies and standards. IEEE Transactions on Industrial Informatics, , 7(4):529-539, 2011.

[10] J. C. Goswami and A. E. Hoefel. Algorithms for estimating instantaneous frequency. Signal Processing, 84(8):1423-1427, 2004.

[11] D. W. Clarke. Designing phase-locked loops for instrumentation applications. Measurement, 32(3):205-227, 2002.

[12] S. C. Gupta. Phase-locked loops. Proceedings of the IEEE, 63(2):291$306,1975$.
[13] W. C. Lindsey and M. K. Simon. Phase-Locked Loops \& Their Application. New York : IEEE Press, 1978.

[14] A. V. Oppenheim and R. W. Schafer. Digital Signal Processing. London : Prentice-Hall International, 1986.

[15] G H Golub and C F Van Loan. Matrix Computations. Baltimore : The Johns Hopkins University Press, 2nd edition, 2013.

[16] T. Sderstrm and P. Stoica. System Identification. New York ; London : Prentice-Hall, 1989.

[17] K. Yoo and H. Park. Fast residual computation for sliding window recursive least squares methods. Signal Processing, 45(1):85-95, 1995.

[18] F. Gustafsson. Adaptive Filtering and Change Detection. Chichester : Wiley, 2000.

[19] K. Koch. Parameter Estimation and Hypothesis Testing in Linear Models. Berlin : Springer-Verlag, 1988. 